\title{
A Systematic Construction of Abstract Domains
}

\author{
Dmitri Boulanger ${ }^{\star} \quad$ Maurice Bruynooghe ${ }^{\star \star}$ \\ Department of Computer Science, Katholieke Universiteit Leuven \\ Celestijnenlaan 200 A, B-3001, Heverlee, Belgium \\ email: $\{$ dmitri, maurice\}@cs.kuleuven.ac.be
}

\begin{abstract}
An abstract domain construction technique for static analysis of definite logic programs is presented. A complete family of preinterpretations, induced by equality theories with non-term elements, is used as a family of abstract domains, allowing all possible levels of precision.
\end{abstract}

\section{Introduction}

The key step in developing an abstract interpretation for logic programs consists of choosing an abstract domain, whose elements are data descriptions, and defining operations over these data descriptions. The most important operation is the unification of data descriptions which should safely approximate standard unification over the "concrete" domain.

When interested in declarative semantics, data descriptions representing sets of ground terms are adequate. Data descriptions such as rigid types [15] and unary regular types [13] have been used. When interested in operational semantics, it is necessary to deal with non-ground terms. To perform unification over such data descriptions with a minimum precision, one has to cope with dependencies between terms. Several domains capturing dependencies have been developed, such as Prop [21], Asub [22], and Sharing [16]. Some sharing component also shows up in domains capturing structural information such as integrated types [15], the pattern domain and the abstract equation systems of $[6,4]$.

This paper takes a different approach. The idea is to partition the universe of terms into a number of equivalence classes. Restricting to ground terms only, the concrete semantics defines equivalence classes which are singletons. The abstract semantics can define larger classes. In a sense, this is is what is being done by the well-known initial algebra technique (see, e.g. $[14,24]$ ). Considering non-ground terms is more complex. To start with, the concrete semantics has equivalence classes which are not singletons, but are sets closed under renaming. The central idea of the paper is the development of machinery to abstract such sets.

\footnotetext{
* Supported by the K.U.Leuven. Permanent address: Keldysh Institute for Applied Mathematics, Russian Academy of Science, Miusskaya sq., 4, 125047 Moscow, Russia.

* Supported by the Belgian National Fund for Scientific Research
} 
The rest of the paper is as follows. Section 2 recalls notions of first order languages and interpretations. Section 3 is the main one: it introduces a class of interpretations of a first order equality language. This class is used as basis for constructing a complete family of abstract domains. Section 4 introduces an abstract interpretation framework for definite logic programs.

\section{Preliminaries: First Order Languages}

Our formalisation of first order languages mostly follows Lloyd [19]. A first order language $\mathcal{L}$ is based on a ranked alphabet $\langle F, P\rangle$, where $F \neq \emptyset$ and $P \neq \emptyset$ ( $F$ and $P$ are function and predicate symbols of assigned arities, respectively), $F \cap P=\emptyset$, and on a countable set of variables $\mathcal{V}$. Terms are constructed from $F$ and $\mathcal{V}$ as usual. Also, $f$ and $p, t, s$ and $\bar{t}, \bar{s}$ denote function and predicate symbols, terms and tuples of terms based on $\mathcal{L}$, respectively.

Given a well-formed formula $F$ based on $\mathcal{L}, \forall: F$ and $\exists: F$ denotes universal and existential closure of $F$, respectively. Also, given variables $X_{1}, \ldots, X_{n} \in \mathcal{V}$, $n>0$, the expression $\left.F\right|_{X_{1}, \ldots, X_{n}}$, called the projection of the formula $F$ onto the variables $X_{1}, \ldots, X_{n}$, is a shorthand for $\exists v-\left\{X_{1}, \ldots, X_{n}\right\}: F$.

Given a syntactic object $O$ based on $\mathcal{L}, \operatorname{var}(O)$ denotes the set of free variables occurring in $O$. Syntactical objects $O$ and $O^{\prime}$ are said to be independent, noted $O$ ind $O^{\prime}$, iff $\operatorname{var}(O) \cap \operatorname{var}\left(O^{\prime}\right)=\emptyset$. We denote by $\bar{O}$ a tuple of pairwise distinct syntactic objects, e.g. $\bar{X}$ denotes a tuple of distinct variables. Given a syntactic object $O$ of $\mathcal{L}, O \cong$ denotes an equivalence class of $O$ wrt the syntactic variance

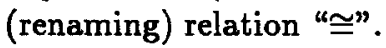

An interpretation $\mathrm{I}$ for a first order language is a triple $\langle\mathrm{D}, \Lambda, \mathcal{H}\rangle$, where $D$ is the domain of the interpretation (a non-empty set), $\Lambda$ is an assignment of mappings of the form $D \times D \times \cdots \times D \mapsto D$ to the function symbols of $F$ and $\mathcal{H}$ is a truth function which maps atoms $p\left(\partial_{1}, \ldots, \partial_{n}\right)$ with $\partial_{1}, \ldots, \partial_{n} \in \mathrm{D}, n \geq 0$, $p^{(n)} \in \mathrm{P}$ to a truth value from $\{\mathbf{f}, \mathrm{t}\}$ where $\mathbf{f}<\mathbf{t}, \mathbf{f}^{-1}=\mathbf{t}$ and $\mathbf{t}^{-1}=\mathbf{f}$. The truth function is determined by the set of atoms mapped to $t$. Given an interpretation $\mathbf{I}=\langle\mathrm{D}, \Lambda, \mathcal{H}\rangle$, the pair $\langle\mathrm{D}, \Lambda\rangle$, noted $\mathrm{I}_{\mathbf{o}}$, is called a pre-interpretation. A Herbrand interpretation of $\mathcal{L}$ has the Herbrand universe, noted $\mathrm{HU}_{\mathcal{L}}$, as domain. Given an interpretation $\mathrm{I}=\langle\mathrm{D}, \Lambda, \mathcal{H}\rangle$ of $\mathcal{L}$ based on $\langle\mathrm{F}, \mathrm{P}\rangle$, we denote by:

- $\partial$ and $\bar{\partial}$ a domain element of $D$ and a tuple of domain elements of $\bar{D}$, respectively ( $\overline{\mathrm{D}}$ denotes the set of tuples of domain elements)

$-\mathbf{I}(p), p^{(n)} \in \mathrm{P}, n \geq 0$ the set $\{p(\bar{\partial}) \mid \bar{\partial} \in \overline{\mathrm{D}}, \mathcal{H}(p(\bar{\partial}))=\mathbf{t}\}$

- I $(f)(\bar{\partial}), f \in F, \bar{\partial} \in \bar{D}$ a domain element $\hat{\partial} \in \mathrm{D}$ such that $f(\bar{\partial}) \mapsto \hat{\partial} \in A$

\subsection{Domain Terms and Domain Formulas}

We borrow some notations from [10]. Given a first order language $\mathcal{L}$ based on alphabet $\langle F, P\rangle$ and its interpretation $I$ with domain $D$, we extend the standard notion of syntactic object of $\mathcal{L}$ by allowing domain elements $\partial$ of $D$ to appear in them. Domain terms / atoms / formulas are terms/atoms/formulas containing elements of $D$. 
The interpretation I induces a unique mapping $\tilde{\mathbf{I}}$ on closed (i.e. ground) domain terms and atoms which is defined as follows: for any $\partial: \tilde{\mathbf{I}}(\partial)=\partial$ and

- for any $f^{(n)} \in \mathrm{F}, n \geq 0: \tilde{\mathbf{I}}\left(f\left(\mathrm{t}_{1}, \ldots, \mathrm{t}_{n}\right)\right)=\mathbf{I}(f)\left(\tilde{\mathbf{I}}\left(\mathrm{t}_{1}\right), \ldots, \tilde{\mathbf{I}}\left(\mathrm{t}_{n}\right)\right)$

- for any $p^{(n)} \in \mathrm{P}, n \geq 0: \tilde{\mathbf{I}}\left(p\left(\mathrm{t}_{1}, \ldots, \mathrm{t}_{n}\right)\right)=p\left(\tilde{\mathbf{I}}\left(\mathrm{t}_{1}\right), \ldots, \tilde{\mathbf{I}}\left(\mathrm{t}_{n}\right)\right)$

where $t_{1}, \ldots, t_{n}$ are closed domain terms based on $\mathcal{L}$ and $I$.

A variable assignment $V$ is a set of tuples $X / t$ with $X$ a variable and $t$ a domain term. Given a syntactic object $O, \mathbf{V}(O)$ denotes the result of a variable assignment, i.e. $\mathbf{V}(O)$ is an instance of $O$. The truth function $\mathcal{H}_{\mathbf{I}}$ of the interpretation $I$ can be extended to all closed domain formulas as follows: for any ground domain atom $p\left(\mathrm{t}_{1}, \ldots, \mathrm{t}_{n}\right): \mathcal{H}_{\mathbf{I}}\left(p\left(\mathrm{t}_{1}, \ldots, \mathrm{t}_{n}\right)\right)=\mathcal{H}_{\mathbf{I}}\left(p\left(\tilde{\mathbf{I}}\left(\mathrm{t}_{1}\right), \ldots, \tilde{\mathbf{I}}\left(\mathrm{t}_{n}\right)\right)\right) ;$ for any closed domain formula $F: \mathcal{H}_{\mathbf{I}}(\neg F)=\mathcal{H}_{\mathbf{I}}(F)^{-1}$ and

$$
\begin{array}{ll}
\mathcal{H}_{\mathbf{I}}(F \vee G)=\max \left\{\mathcal{H}_{\mathbf{I}}(F), \mathcal{H}_{\mathbf{I}}(G)\right\} & \mathcal{H}_{\mathbf{I}}(F \wedge G)=\min \left\{\mathcal{H}_{\mathbf{I}}(F), \mathcal{H}_{\mathbf{I}}(G)\right\} \\
\mathcal{H}_{\mathbf{I}}(F \leftrightarrow G)=\mathcal{H}_{\mathbf{I}}(F \wedge G \vee \neg \wedge \neg) & \mathcal{H}_{\mathbf{I}}(F \leftarrow G)=\mathcal{H}_{\mathbf{I}}(F \vee \neg G) \\
\mathcal{H}_{\mathbf{I}}(\exists X: F)=\max \left\{\mathcal{H}_{\mathbf{I}}(\{X / \partial\}(F)) \mid \partial \in \mathrm{D}\right\} & \\
\mathcal{H}_{\mathbf{I}}\left(\forall \forall_{X}: F\right)=\min \left\{\mathcal{H}_{\mathbf{I}}(\{X / \partial\}(F)) \mid \partial \in \mathrm{D}\right\} &
\end{array}
$$

This extension of the truth function enables us to consider models of closed domain formulas.Given an interpretation $I$ and a closed (domain) formula $F$, we write $\mathbf{I} \models F$ iff $\mathcal{H}_{\mathbf{I}}(F)=\mathbf{t}$. This extends to $\mathbf{I} \models \mathcal{T}, \mathcal{T}$ being a theory in the classical way. A model of $\mathcal{T}$ is denoted by $\mathbf{M}_{\mathcal{T}}$. Notice that the interpretation of closed formulas without domain elements is unchanged.

\subsection{Equality Theories}

Let $\mathcal{L}$ be a first order language with equality, i.e. the set of predicate symbols $\mathrm{P}$ of $\mathcal{L}$ includes the equality predicate ${ }^{(2)}(\mathcal{L}$ is said to be an equality language if $\mathrm{P}$ only consists of $\left.=^{(2)}\right)$. An equality theory based on $\mathcal{L}$ is a first order theory based on $\mathcal{L}$ such that the only predicate symbol occurring in it is the equality predicate. Given a pair of tuples of terms $\left\langle\mathrm{t}_{1}, \ldots, \mathrm{t}_{n}\right\rangle$ and $\left.\left\langle\mathrm{s}_{1}, \ldots, \mathrm{s}_{n}\right\rangle, n\right\rangle 0$, noted $\overline{\mathrm{t}}$ and $\overline{\mathrm{s}}$ respectively, we denote by $\overline{\mathrm{t}}=\overline{\mathrm{s}}$ the equality formula $\mathrm{t}_{1}=\mathrm{s}_{1} \wedge \cdots \wedge \mathrm{t}_{n}=\mathrm{s}_{n}$.

The standard Equality theory of $\mathcal{L}$, noted $E Q(\mathcal{L})$, is as follows:

Equivalence Axioms

$\forall: X=X$

$\forall: X=Y \leftarrow Y=X$

$\forall: X=Z \leftarrow X=Y \wedge Y=Z$

\section{Congruence(Substitutivity) Axioms}

for any $p \in \mathrm{P}: \quad \forall: p(\bar{X}) \leftarrow p(\bar{Y}) \wedge \bar{X}=\bar{Y}$

for any $f \in \mathrm{F}: \quad \forall: f(\bar{X})=f(\bar{Y}) \leftarrow \bar{X}=\bar{Y}$

Given an interpretation I of $\mathcal{L}$ with domain D satisfying the axioms of $E Q(\mathcal{L})$ ( $I$ is a model of $E Q(\mathcal{L})$ ), the equality predicate is an equivalence relation over elements of the domain $D$. This equivalence relation forms a congruence for all predicates and functions of $\mathcal{L}$. Interpreting $=^{(2)}$ as identity, i.e. $\mathbf{I}(=)=$ $\{\partial=\partial \mid \partial \in \mathrm{D}\}$, one obtains a model of $E Q(\mathcal{L})$. This is done in the sequel. 
The second important equality theory is the Free Equality theory (or Clarks Equality Theory), noted $F E Q(\mathcal{L})$. It extends $E Q(\mathcal{L})$ with:

for any function $f^{(n)} \in \mathrm{F}, n>0$ :

for any functions $f, g \in \mathrm{F}$ such that $f \neq g$ :

for any term $t$ of $\mathcal{L}$ containing the variable $X$ :

$$
\begin{aligned}
& \forall: f(\bar{X})=f(\bar{Y}) \rightarrow \bar{X}=\bar{Y} \\
& \forall: \neg(g(\bar{X})=f(\bar{Y})) \\
& \forall: \neg(t=X)
\end{aligned}
$$

The Free Equality axioms formalise unification [19]; it is well-known (e.g., [10]) that $F E Q(\mathcal{L})$ implies the unification algorithm of Martelli and Montanari [20, 17]. A Herbrand interpretation satisfies $F E Q$-axioms if the equality predicate $"={ }^{(2) "}$ is interpreted as (syntactic) identity over the Herbrand universe.

The third equality theory is not so well-known, it is the Non-Term Equality Theory with at least $k$ non-term elements (see, for example, $[23]^{3}$ ), noted $N T E_{k}(\mathcal{L}), k>0$. It extends $E Q(\mathcal{L})$ with:

$$
\exists x_{1}, \cdots, X_{k}\left(\bigwedge_{j=1}^{k} \operatorname{NTE}\left(X_{j}\right)_{\{i \neq j \mid i, j=1, \ldots, k\}} \neg\left(X_{i}=X_{j}\right)\right)
$$

where $\operatorname{NTE}(X)$ denotes the formula $\forall_{\bar{Y}_{1}, \ldots, \bar{Y}_{n}} \bigwedge_{i=1}^{n} \neg\left(X=f_{i}\left(\bar{Y}_{i}\right)\right)$ with $f_{i} \in \mathrm{F}$, $i=1, \ldots, n$ are all function symbols of $\mathcal{L}$ and $\bar{Y}_{i}$ are the corresponding tuples of pairwise distinct variables. $N T E_{k}, k>0$ states that there exist at least $k$ objects which are not terms, i.e. domain elements which are not in the range of any function (in section 3 this property is exploited to represent variables).

Following [23], the equality theories $E Q, F E Q, N T E_{k}$ and $N T E_{\infty}$ (the latter denotes the set $\left\{N T E_{1}, N T E_{2}, \ldots\right\}$ ) are used as a convenient toolkit to characterise pre-interpretations.

\subsection{Isomorphism of Interpretations of a First Order Language}

Given two interpretations $\mathbf{I}=<\mathrm{D}, \Lambda, \dot{\mathcal{H}}>$ and $\mathbf{I}^{\prime}=\left\langle\mathrm{D}^{\prime}, \Lambda^{\prime}, \mathcal{H}^{\prime}>\right.$ of a first order language $\mathcal{L}$, a homomorphism $\mathrm{h}$ from $\mathbf{I}$ onto $\mathrm{I}^{\prime}$, noted $\mathrm{h}: \mathbf{I} \rightarrow \mathrm{I}^{\prime}$, is a mapping "onto" $h: D \rightarrow D^{\prime}$ which satisfies the following conditions:

$-\mathrm{h}(\mathbf{I}(f)(\bar{\partial}))=\mathbf{I}^{\prime}(f)(\mathrm{h}(\bar{\partial}))$ for any function symbol $f$ of $\mathcal{L}$ and any $\bar{\partial} \in \overline{\mathrm{D}}$

- I $\vDash p(\bar{\partial})$ implies $\mathbf{I}^{\prime} \vDash p(h(\bar{\partial}))$ for any predicate symbol $p$ of $\mathcal{L}$ and any $\bar{\partial} \in \overline{\mathrm{D}}$

Let $\mathcal{I}$ denote a class (set) of interpretations of $\mathcal{L}$. Given the corresponding family $\Re$ of homomorphisms "onto" between interpretations of $\mathcal{I}$, the pair < $\mathcal{I}, \Re>$ is a category iff it satisfies the following natural conditions (see [18]):

- for any interpretation $\mathbf{I} \in \mathcal{I}$ there exists a homomorphism $\mathrm{h}: \mathbf{I} \rightarrow \mathbf{I}$ of $\Re$ which is the identity mapping over the domain of $\mathbf{I}$, noted $\epsilon_{\mathbf{I}}$

- if there is a pair $h^{\prime}: I^{\prime} \rightarrow I$ and $h^{\prime \prime}: I \rightarrow I^{\prime \prime}$ of homomorphisms of $\Re$ such that $\mathbf{I}^{\prime}, \mathbf{I}, \mathbf{I}^{\prime \prime} \in \mathcal{I}$, then there exist a homomorphism $\mathrm{h}: \mathbf{I}^{\prime} \rightarrow \mathbf{I}^{\prime \prime}$, noted $\mathrm{h}^{\prime \prime} \circ \mathrm{h}^{\prime}$; the homomorphism $h=h^{\prime \prime} \circ h^{\prime}$ is called the composition of $h^{\prime \prime}$ and $h^{\prime}$

3 In [23] the non-term domain elements are called "roots" or isolated elements. 
- the composition of homomorphisms is associative, i.e. given homomorphisms $h_{1}, h_{2}$ and $h_{3}$ of the family $\Re$, holds: $h_{1} \circ\left(h_{2} \circ h_{3}\right)=\left(h_{1} \circ h_{2}\right) \circ h_{3}$

- if there is a pair $h^{\prime}: I^{\prime} \rightarrow I$ and $h^{\prime \prime}: I \rightarrow I^{\prime \prime}$ of homomorphisms of $\Re$ such that $\mathbf{I}^{\prime}, \mathbf{I}, \mathbf{I}^{\prime \prime} \in \mathcal{I}$, then $h^{\prime \prime} \circ \epsilon_{\mathbf{I}}=h^{\prime \prime}$ and $\epsilon_{\mathbf{I}} \circ h^{\prime}=h^{\prime}$

Let $\mathcal{L}$ be a first order language and $\mathcal{I}$ be a class of interpretations of $\mathcal{L}$. Suppose that the pair $\langle\mathcal{I}, \Re\rangle$ forms a category with appropriate homomorphisms $\Re$. The interpretations $\mathrm{I}$ and $\mathrm{I}^{\prime}$ of $\mathcal{L}$ are said to be isomorphic iff there exists a pair of homomorphisms $\mathrm{h}: \mathbf{I} \rightarrow \mathbf{I}^{\prime}$ and $h^{\prime}: \mathbf{I}^{\prime} \rightarrow \mathbf{I}$ of the family $\Re$ such that $h^{\prime} \circ h=\epsilon_{\mathbf{I}}$ and $h \circ h^{\prime}=\epsilon_{\mathbf{I}}^{\prime}$.

\section{An Abstract Domain Construction}

This section introduces important notions, which form the backbone of our approach. The "concrete" domain is a set of equivalence classes of terms wrt renaming. It can be induced as a model of $F E Q \cup N T E_{\infty}$. The abstraction is achieved by considering weaker equality theories.

\subsection{Complete Lattice of nte-Interpretations}

Let $\mathcal{L}$ be a first order equality language. Suppose that $\mathbf{I}$ is an interpretation of $\mathcal{L}$ with domain $\mathrm{D}$ such that $\mathrm{I} \vDash N T E_{k}(\mathcal{L}), k>0$, i.e. the domain $\mathrm{D}$ of $\mathrm{I}$ contains at least $k$ non-term elements. The latter allows to extend the mapping $\tilde{\mathbf{I}}$ to all domain terms of $\mathcal{L}$ (i.e. to non-ground terms, cf. section 2.1) as follows: given a variable $X \in \mathcal{V}$, we define $\tilde{\mathbf{I}}(X)=v$, where $v \in \mathrm{D}$ is one of the nonterm domain elements. We assume that given an interpretation with non-term domain elements, the mapping of variables $\mathcal{V}$ to the non-term elements is a mapping "onto". For instance, with $\mathcal{V}=\left\{X_{0}, X_{1}, X_{2}, \ldots\right\}$ and non-term elements $\left\{v_{0}, v_{1}, v_{2}, \ldots, v_{k-1}\right\}$, the variable $X_{i}$ is mapped to $v_{i \bmod k}$.

Definition 1. nte-Interpretation

An interpretation $\mathbf{I}=\langle\mathrm{D}, \Lambda, \mathcal{H}\rangle$ of an equality language $\mathcal{L}$ is called a nteinterpretation provided that: (1) $\mathbf{I} \models N T E_{k}(\mathcal{L}), k>0$ and (2) $\partial \in \mathrm{D}$ iff there exists a term $\mathrm{t}$ of $\mathcal{L}$ such that $\tilde{\mathbf{I}}(\mathrm{t})=\partial$.

The above definition introduces two important conditions. The first one is a logical condition, which states that any nte-interpretation is a model of a nonterm equality theory. The second condition is a very strict non-logical domain closure axiom, which regulates existence of the domain element: any domain element is "justified" by being the interpretation of a term.

Given a $n t e$-interpretation $I$ of $\mathcal{L}$, def. 1 enables us to define the mapping $\tilde{\mathbf{T}}: D \rightarrow \wp \operatorname{Terms}(\mathcal{L})$, which is the reverse of the mapping $\tilde{\mathbf{I}}$, as follows: for any domain element $\partial \in \mathrm{D}: \tilde{\mathbf{T}}(\partial)=\{\mathrm{t} \in \operatorname{Terms}(\mathcal{L}) \mid \tilde{\mathbf{I}}(\mathrm{t})=\partial\}$. This can be extended to tuples in a natural way: $\tilde{\mathbf{T}}(\bar{\partial})=\{\overline{\mathrm{t}} \in \overline{\operatorname{Terms}}(\mathcal{L}) \mid \tilde{\mathbf{I}}(\overline{\mathrm{t}})=\bar{\partial}\}$, where $\overline{\text { Terms }}(\mathcal{L})$ is the set of tuples of terms based on $\mathcal{L}$ and given a tuple of terms $\overline{\mathbf{t}}=\left\langle\mathrm{t}_{1}, \ldots, \mathrm{t}_{n}\right\rangle, n>0$, we define $\tilde{\mathbf{I}}(\overline{\mathbf{t}})=\left\langle\tilde{\mathbf{I}}\left(\mathrm{t}_{1}\right), \ldots, \tilde{\mathbf{I}}\left(\mathrm{t}_{n}\right)>\right.$. 
Example 1. Consider an equality language $\mathcal{L}$, whose alphabet consists of a set of function symbols $F=\left\{[]^{(0)}, a^{(0)}, s(-)^{(1)},\left[-H_{-}\right]^{(2)}\right\}$ and a set of predicate symbols $\mathrm{P}=\left\{=^{(2)}\right\}$. Let $\mathrm{I}_{\mathbf{0}}=\langle\mathrm{D}, \Lambda>$ be the following pre-interpretation of $\mathcal{L}$ : the domain $\mathrm{D}$ of the interpretation is the set $\{v, \gamma, \iota\}$ and the mapping $\Lambda$ assigned to the function symbols of $F$ is defined by:

$$
\begin{array}{lllllll}
a \mapsto \gamma & {[\gamma \mid \gamma] \mapsto \gamma} & {[v \mid *] \mapsto \iota} & {[\iota \mid *] \mapsto \iota} & s(v) & \mapsto \iota \\
{[] \mapsto \gamma} & s(\gamma) \mapsto \gamma & {[* \mid v] \mapsto \iota} & {[* \mid \iota] \mapsto \iota} & s(\iota) & \mapsto \iota
\end{array}
$$

where " $*$ " denotes any element of $D$. Notice that $v$ is a unique non-term element. Also, observe that if $\mathbf{I}=\mathbf{I}_{\mathbf{0}} \cup \mathbf{I}(=)$, where " $=$ " is an identity over $D$, then $\mathbf{I} \models N T E_{1}(\mathcal{L})$ and it is a nte-interpretation of $\mathcal{L}$. Indeed, we have that $\tilde{\mathbf{T}}(\gamma)=$ $\{[, a, s(0), \ldots\}$, i.e. it is the set of all ground terms, $\tilde{\mathbf{T}}(v)=\mathcal{V}$, and $\tilde{\mathbf{T}}(\iota)$ is the set of all remaining terms. In the sequel this interpretation is noted $I^{e x 1}$.

A nte-interpretation of $\mathcal{L}$ is called minimal, noted $\mathbf{I}^{\mathrm{min}}$, if its domain $\mathrm{D}^{\mathrm{min}}$ contains the minimal number of elements. Also, an nte-interpretation is called maximal, noted $I^{\text {max }}$, provided that for any pair of domain elements $\partial, \partial^{\prime}$ such that $\partial \neq \partial^{\prime}$ holds: $\tilde{\mathbf{I}}(\mathrm{t}) \neq \tilde{\mathbf{I}}\left(\mathrm{t}^{\prime}\right)$ iff $\mathrm{t} \not \equiv \mathrm{t}^{\prime}$, where $\mathrm{t}$ and $\mathrm{t}^{\prime}$ are terms of $\mathcal{L}$ and " $\equiv$ " denotes syntactical identity. The domain closure axiom combined with this condition implies that the domain $D^{\max }$ of $\mathbf{I}^{\max }$ contains the maximal number of elements. The latter is exactly the number of terms in $\mathcal{L}$.

The domain $\mathrm{D}^{\text {min }}$ of the minimal nte-interpretation $\mathrm{I}^{\text {min }}$ of $\mathcal{L}$ consists of two domain elements, i.e. it is the set $\{v, \partial\}$, where the domain element $v$ is a unique non-term element and $\partial$ is such that for any non-variable term $t$ of $\mathcal{L}: \tilde{\mathbf{I}}^{\mathrm{min}}(\mathrm{t})=$ a. A minimal nte-interpretation is a model of the most weak non-term equality theory, i.e. $\mathbf{I}^{\mathrm{min}} \models N T E_{1}(\mathcal{L})$. Def. 1 implies that $\mathbf{I}^{\max }$ is a model of the most strong (precise) non-term equality theory, i.e. $\mathrm{I}^{\max } \vDash F E Q(\mathcal{L}) \cup N T E_{\infty}(\mathcal{L})$.

Given an equality language $\mathcal{L}$ based on functions $F, \mathcal{I}^{\text {nte }}(\mathcal{L})$ denotes the class of all nte-interpretations of $\mathcal{L}$. We can only consider the corresponding set of all pre-interpretations $\mathcal{I}_{\mathbf{0}}{ }^{\text {nte }}(\mathcal{L})$ because the equality predicate is always interpreted as identity. Using an algebraic specification setting, the pre-interpretations of $\mathcal{I}_{\mathrm{o}}{ }^{\text {nte }}(\mathcal{L})$ form a class of one-sorted $\Sigma$-algebras, where the signature $\Sigma$ is the set of functions $F$ (see, e.g. [24]).

\section{Definition 2. Preorder over nte-Interpretations}

Given two pre-interpretations $\mathbf{I}_{\mathbf{o}}, \mathbf{I}_{\mathbf{o}}{ }^{\prime} \in \mathcal{I}_{\mathbf{o}}{ }^{\text {nte }}(\mathcal{L})$ having domains $\mathrm{D}$ and $\mathrm{D}^{\prime}$, respectively, $\mathbf{I}_{\mathbf{0}}{ }^{\prime}$ is said to be more precise than $\mathbf{I}_{\mathbf{o}}$, noted $\mathbf{I}_{\mathbf{0}} \preceq \mathbf{I}_{\mathbf{0}}{ }^{\prime}$, iff there exists a homomorphism $h: \mathbf{I}_{\mathbf{o}}^{\prime} \rightarrow \mathbf{I}_{\mathbf{0}}$ which is defined by: given $\partial \in D$ and $\partial^{\prime} \in D^{\prime}, h\left(\partial^{\prime}\right)=\partial$ iff $\tilde{\mathbf{T}}^{\prime}\left(\partial^{\prime}\right) \subseteq \tilde{\mathbf{T}}(\partial)$.

As usual (see $[14,24]$ ), the above definition implies that the pair $\left\langle\mathcal{I}_{\mathbf{o}}^{\text {nte }}, \Re^{\text {nte }}\right\rangle$ forms a category with the family $\Re^{n t e}$ of homomorphisms between nte-interpretations. Also, $\mathbf{I}_{\mathbf{o}} \preceq \mathbf{I}_{\mathbf{o}}{ }^{\prime}$ and $\mathbf{I}_{\mathbf{o}}{ }^{\prime} \preceq \mathbf{I}_{\mathbf{0}}$ iff the pre-interpretations $\mathbf{I}_{\mathbf{o}}$ and $\mathbf{I}_{\mathbf{o}}{ }^{\prime}$ are isomorphic. It is clear that for any $\mathbf{I}_{\mathbf{0}} \in \mathcal{I}_{\mathbf{0}}{ }^{\text {nte }}(\mathcal{L}): \mathbf{I}_{\mathbf{0}}{ }^{\min } \preceq \mathbf{I}_{\mathbf{0}} \preceq \mathbf{I}_{\mathbf{0}}{ }^{\max }$. Therefore, the category $<\mathcal{I}_{\mathbf{o}}{ }^{\text {nte }}, \Re^{n t e}>$ has the pre-interpretations ( $\Sigma$-algebras) $\mathbf{I}_{\mathbf{0}}{ }^{\max } / \mathbf{I}_{\mathbf{0}}{ }^{\min }$ as initial/terminal, respectively. The initiality of $\mathbf{I}^{\text {max }}$ implies 
that $\tilde{\mathbf{I}}^{\max }$ is an isomorphism between Terms and $\mathrm{D}^{\max }$. The set $\left\langle\mathcal{I}_{\mathrm{o}}{ }^{\text {nte }}(\mathcal{L}), \preceq>\right.$ is a partially ordered set provided that isomorphic pre-interpretations are not distinguishable. The latter is always assumed in the sequel, and, therefore, the partially ordered set $\left\langle\mathcal{I}_{\mathbf{o}}{ }^{\text {nte }}(\mathcal{L}), \preceq\right\rangle$ is a complete lattice [24].

\subsection{An Abstract Domain}

The existence of a preorder over tuples of terms of $\mathcal{L}$ is crucial in our setting.

\section{Definition 3. Preorder over Tuples of Terms modulo nte-Interpretation}

Consider an equality language $\mathcal{L}$ and $n t e$-interpretation $\mathbf{I}$ of $\mathcal{L}$. Given two tuples of terms $\overline{\mathrm{t}}$ and $\overline{\mathrm{s}}$ of $\operatorname{Terms}(\mathcal{L})$ having the same arity, the tuple $\overline{\mathrm{t}}$ is said to be more general than the tuple $\overline{\mathrm{s}}(\overline{\mathrm{s}}$ is an instance of $\overline{\mathrm{t}}$ ) modulo nte-interpretation $\mathbf{I}$, noted $\overline{\mathrm{t}} \underline{\mathrm{I}}_{\mathbf{I}} \overline{\mathbf{s}}$, iff $\mathbf{I} \vDash \forall:\left.\left.(\bar{X}=\overline{\mathrm{t}})\right|_{\bar{X}} \leftarrow(\bar{X}=\overline{\mathrm{s}})\right|_{\bar{X}}$.

The above definition relies on the following important concept, which is central to our approach. Suppose that $D$ is the domain of $I$. Given a tuple of terms $\overline{\mathrm{t}}$ of $\mathcal{L}$, such that $\operatorname{var}(\overline{\mathrm{t}})=\bar{U}$, its domain representation, noted $\mathcal{D}_{\mathbf{I}}(\overline{\mathrm{t}})$, is a set of tuples of domain elements, defined as $\mathcal{D}_{\mathbf{I}}(\overline{\mathfrak{t}})=\{\bar{\partial} \in \bar{D} \mid \mathbf{I} \models \exists: \bar{\partial}=\overline{\mathrm{t}}\}$, i.e. the set of tuples $\bar{\partial}$ is obtained by interpreting $\overline{\mathrm{t}}$ under all possible assignments of domain elements to the variables $\bar{U}$. This means that the tuple $\overline{\mathrm{t}}$ is considered as a function over the domain $D$ of $\mathbf{I}$ with the variables $\bar{U}$ as the "formal parameters" (ground tuples correspond to constants and tuples constructed from distinct variables are identity functions). From this point of view, $\mathcal{D}_{\mathbf{I}}(\overline{\mathrm{t}})$ is the set off all targets of this function (more details in section 3.3). For instance, in the context of example 1: $\mathcal{D}_{\mathbf{I}=x \mathbf{1}}(U)=\{v, \iota, \gamma\}, \mathcal{D}_{\mathbf{I}=x_{1}}(s(U))=\{\iota, \gamma\}, \mathcal{D}_{\mathbf{I e x 1}}(a)=\{\gamma\}$.

Def. 3 immediately implies that given tuples of terms $\overline{\mathrm{t}}$ and $\overline{\mathrm{s}}$, holds: $\overline{\mathrm{t}} \sqsubseteq_{\mathbf{I}} \overline{\mathbf{s}}$ iff $\mathcal{D}_{\mathbf{I}}(\overline{\mathrm{t}}) \supseteq \mathcal{D}_{\mathbf{I}}(\overline{\mathrm{s}})$. On the other hand, we have that $\bar{\partial} \in \mathcal{D}_{\mathbf{I}}(\overline{\mathrm{t}})$, iff there exists a variable assignment (substitution) $\mathbf{V}=\{\bar{U} / \overline{\mathrm{s}}\}$, where $\operatorname{var}(\overline{\mathrm{t}})=\bar{U}$ and $\overline{\mathrm{s}}$ is a tuple of terms, such that: $\tilde{\mathbf{I}}(\{\bar{U} / \bar{s}\}(\overline{\mathrm{t}}))=\bar{\partial}$ with $\tilde{\mathbf{I}}$ extended to variables (see section 2.1 and 3.1). Recall that $\tilde{\mathbf{I}}^{\text {max }}$ is an isomorphism between Terms and $\mathrm{D}^{\max }$. This means that the relation "ㄷmax" is the standard syntactic relation "more general than" over tuples of terms because the set $\mathcal{D}_{\operatorname{Imax}}(\overline{\mathfrak{t}})$ is the set of all syntactic instances of $\overline{\mathfrak{t}}$. Therefore, def. 3 generalises the standard syntactic notion and the proposition below gives a natural way to abstract it:

Proposition 4. Consider an equality language $\mathcal{L}$. Let $\mathrm{I}^{\max }$ be the maximal nteinterpretation and $\mathbf{I}, \mathbf{I}^{\prime}$ be a pair of nte-interpretations of $\mathcal{L}$. Given a pair of tuples of terms $\overline{\mathrm{t}}$ and $\overline{\mathrm{s}}$ based on $\mathcal{L}$, holds: (1) $\overline{\mathrm{s}}$ is a syntactic instance of $\overline{\mathrm{t}}$ iff

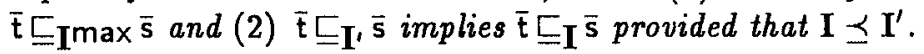

The equivalence relation modulo nte-interpretation $I$ is induced as usual: $\overline{\mathrm{t}} \approx_{\mathbf{I}} \overline{\mathrm{s}}$ iff $\overline{\mathrm{t}} \underline{\mathbf{I}}_{\mathbf{I}} \overline{\mathrm{s}}$ and $\overline{\mathrm{s}} \underline{E}_{\mathbf{I}} \overline{\mathrm{t}}$, i.e. the standard syntactic variance relation (renaming) is also generalised by def.3. For instance, in the context of example 1: $[X, Y] \sqsubseteq_{I}$ max $[Z, Z]$ and $[X, Y] \approx_{I_{\Phi \times 1}}[Z, Z] ;$ however, $\langle X, Y\rangle \Xi_{I_{\text {ex } 1}}\langle Z, Z\rangle$ (the tuples $\langle X, Y\rangle$ and $\langle Z, Z\rangle$ are not equivalent wrt $I^{\operatorname{exI}}$ but the lists $[X, Y]$ and $[Z, Z]$ are). 
Proposition 4 shows that the syntactic notions of renaming and instance, which form the basis in the standard (syntactic) concepts of unification and of most general unifier [11], can equivalently be represented and abstracted using nte-interpretations because $\mathbf{I} \preceq I^{\max }$ for any nte-interpretation $\mathbf{I}$. This is the basis of the abstraction technique developed below in section 4 .

Any nte-interpretation $\mathbf{I}$ of $\mathcal{L}$ induces a set of equivalence classes of tuples of terms modulo I, noted $\Upsilon_{\mathbf{I}}(\mathcal{L})$. The set of equivalence classes modulo I of tuples of terms having arity $k>0$, noted $\Upsilon_{\mathrm{I}}^{(k)}$, is a partially ordered set, noted < $\Upsilon_{\mathbf{I}}{ }^{(k)}$, ㄷ․ $_{\mathbf{I}}>$, having the most general element, which is the equivalence class of tuples only constructed from distinct variables. In the sequel the equivalence classes (types) of $\Upsilon_{\mathbf{I}}$ are denoted $\tau_{1}, \ldots, \tau_{n}, \ldots$ For instance, the nte-interpretation $\mathbf{I}^{e x 1}$ of example 1 induces the set of types of terms $\Upsilon_{\mathbf{I}=\infty 1}^{(1)}=\left\{\tau_{v}^{e x 1}, \tau_{b}^{e x 1}, \tau_{\gamma}^{e x 1}\right\}$, where: $\tau_{v}^{e x 1}$ is the equivalence class of all variables; $\tau_{t}^{e x 1}$ is the equivalence class of all non-ground instantiated terms; $\tau_{\gamma}^{\text {ex } 1}$ is the equivalence class of ground terms. Notice that $\tau_{v}^{e x 1} \sqsubseteq_{I=x 1} \tau_{b}^{e x 1} \sqsubseteq_{I=x+1} \tau_{\gamma}^{e x 1}$.

In what follows the family of posets $\left\langle\Upsilon_{\mathbf{I}}^{(k)}, \Xi_{\mathbf{I}}\right\rangle, \Upsilon_{\mathbf{I}}=\cup_{k=1}^{\infty} \Upsilon_{\mathbf{I}}^{(k)}$ is considered as an abstract domain. The total mapping "onto" $\mathrm{H}: \overline{\text { Terms }} \cong \rightarrow \Upsilon_{\mathrm{I}}$, defined as $\mathrm{H}\left(\overline{\mathrm{t}}^{\approx}\right)=\tau$ iff $\overline{\mathrm{t}} \in \tau$, plays the role of an abstraction function induced by the nte-interpretation $I$. The next section discusses the properties of this abstraction function.

\subsection{Abstracting Syntactic Variance: An Initial Algebra Approach}

We can define the domain representation for any $\tau \in \Upsilon_{\mathbf{I}}$ as follows: $\mathcal{D}_{\mathbf{I}}(\tau)=$ $\mathcal{D}_{\mathbf{I}}(\overline{\mathrm{t}})$, where $\overline{\mathrm{t}}$ is an arbitrary tuple of terms such that $\overline{\mathrm{t}} \in \tau$. It is not difficult to see that $\tau \sqsubseteq_{\mathbf{I}} \tau^{\prime}$ iff $\mathcal{D}_{\mathbf{I}}(\tau) \supseteq \mathcal{D}_{\mathbf{I}}\left(\tau^{\prime}\right)$, where $\tau, \tau^{\prime} \in \Upsilon_{\mathbf{I}}$. For instance, in the context of example 1 , we have $\mathcal{D}_{\mathbf{I}^{e x 1}}\left(\tau_{v}^{e x 1}\right)=\{v, \iota, \gamma\}, \mathcal{D}_{\mathbf{I}^{e x 1}}\left(\tau_{\iota}^{e x 1}\right)=\{\iota, \gamma\}$, $\mathcal{D}_{\text {Iox1 }}\left(\tau_{\gamma}^{e x 1}\right)=\{\gamma\}$. Notice that not all sets of domain elements represent a type - the set $\{v, \gamma\}$ does not correspond to any type of $\Upsilon_{I=x 1}$.

Proposition 5. Let $\overline{\mathrm{t}}, \operatorname{var}(\overline{\mathrm{t}})=\bar{X}^{(k)}, k>0$, be a tuple of terms of $\mathcal{L}$ having arity $n>0$. Given a type $\tau \in \Upsilon_{I}^{(k)}$, there exists a unique type $\hat{\tau} \in \Upsilon_{I}^{(n)}$ such that $\mathcal{D}_{\mathbf{I}}(\hat{\tau})=\left\{\tilde{\mathbf{I}}(\{\bar{X} / \bar{\partial}\}(\overline{\mathrm{t}})) \mid \bar{\partial} \in \mathcal{D}_{\mathbf{I}}(\tau)\right\}=\mathcal{D}_{\mathbf{I}}(\{\bar{X} / \overline{\mathbf{s}}\}(\overline{\mathrm{t}}))$ for any tuple $\overline{\mathbf{s}} \in \tau$.

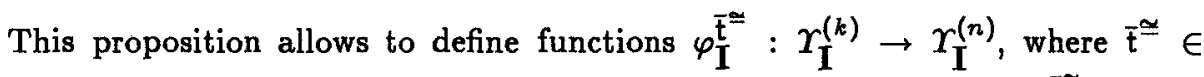
$\overline{\text { Terms }} \cong$ such that $\operatorname{var}(\overline{\mathrm{t}}) \neq \emptyset$, as follows: for any $\tau \in \Upsilon_{\mathbf{I}}^{(k)}$ : the type $\varphi_{\mathbf{I}}^{\hat{\mathrm{t}}^{\tilde{z}}}(\tau)=\hat{\tau}$, $\hat{\tau} \in \Upsilon_{\mathbf{I}}^{(n)}$, is defined by $\mathcal{D}_{\mathbf{I}}(\hat{\tau})=\left\{\tilde{\mathbf{I}}(\{\bar{X} / \bar{\partial}\}(\overline{\mathfrak{t}})) \mid \bar{\partial} \in \mathcal{D}_{\mathbf{I}}(\tau)\right\}$. Ground tuples $\overline{\mathrm{t}}$ having arity $n$ induce functions $\varphi_{\mathbf{I}}^{\overline{\mathrm{t}}}=\rightarrow \Upsilon_{\mathbf{I}}^{(n)}$, which are constants, defined by $\varphi_{\overline{\mathbf{I}}}^{\overline{\mathrm{t}}} \bar{\approx}=\tilde{\mathbf{I}}(\overline{\mathrm{t}})$. The constants have no arguments; all other functions are unary. These functions are indexed or generated by the elements of Terms $\cong$.

Using an algebraic specification setting, the set of quotient tuples Terms $\cong$ defines a sensible many-sorted signature $\Sigma$ (see, e.g. $[14,24]$ ) with a countable 
set of sorts which are arities of tuples of terms. Each element (index) $\overline{\mathrm{t}} \simeq$ of the signature $\Sigma=\overline{\text { Terms }}$ has a type (sort) denotation $k \mapsto n$ with $k>0$ the sort of the argument (number of distinct variables in $\bar{t}$ ) and with $n>0$ the sort of the target (arity of $\overline{\mathrm{t}}$ ). The above functions, noted $\Phi_{\mathrm{I}}$, is a many-sorted $\Sigma$-algebra with the many-sorted domain $\Upsilon_{\mathbf{I}}$. This implies that all functions of $\Phi_{\mathbf{I}}$ are total. Propositions 4 and 5 imply that the functions of $\Phi_{I}$ are monotonic.

The set $\left\{\Phi_{\mathbf{I}} \mid \mathbf{I} \in \mathcal{I}^{\text {nte }}(\mathcal{L})\right\}$ forms a category of monotonic $\Sigma$-algebras with a family of monotonic homomorphisms $\mathrm{H}$ defined by the proposition below:

Proposition 6. Given two $\Sigma$-algebras $\Phi_{\mathbf{I}}$ and $\Phi_{\mathbf{I}^{\prime}}$, such that $\mathbf{I} \preceq \mathbf{I}^{\prime}$, there exists the unique mapping "onto" $\mathrm{H}: \Upsilon_{\mathbf{I}}^{\prime} \rightarrow \Upsilon_{\mathbf{I}}$ satisfying the following conditions:

- for any $\tau \in \Upsilon_{\mathbf{I}}$ and $\tau^{\prime} \in \Upsilon_{\mathbf{I}^{\prime}}: \mathrm{H}\left(\tau^{\prime}\right)=\tau$ iff $\tilde{\mathbf{T}}^{\prime}\left(\tau^{\prime}\right) \subseteq \tilde{\mathbf{T}}(\tau)$

- for any tuple (index) $\tilde{\mathrm{t}}^{\simeq} \in \overline{\text { Terms }}^{\simeq}$ with $k>0$ distinct variables, holds:

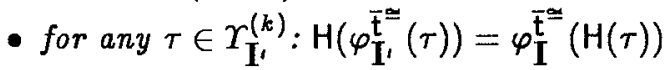

- for any $\tau_{1}, \tau_{2} \in \Upsilon_{\mathbf{I}^{\prime}}^{(k)}: \tau_{1} \sqsubseteq_{\mathbf{I}^{\prime}} \tau_{2}$ implies $\mathrm{H}\left(\tau_{1}\right) \sqsubseteq_{\mathbf{I}} \mathrm{H}\left(\tau_{2}\right)$

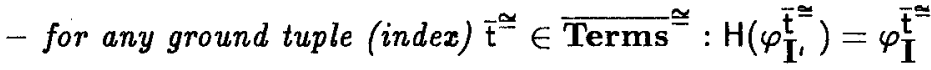

The family of monotonic homomorphisms, noted $H: \Phi_{I^{\prime}} \rightarrow \Phi_{\mathbf{I}}$, between monotonic $\Sigma$-algebras, form a construction which is an instance of the so-called continuous initial algebras approach of [14]: the $\Sigma$-algebra $\Phi_{\mathrm{I} \max }$ is isomorphic with $\overline{\text { Terms }} \simeq$ and it is a monotonic initial algebra; the $\Sigma$-algebra $\Phi_{\mathbf{I} \text { min }}$ is terminal in this class. Given nte-interpretation $\mathrm{I}$, the mapping $\mathrm{H}: \overline{\text { Terms }} \simeq \rightarrow \Upsilon_{\mathrm{I}}$ suggests the following representation of types: a type $\tau \in \Upsilon_{I}$ is defined by a tuple of terms $\overline{\mathrm{t}}$ such that $\mathrm{H}\left(\overline{\mathrm{t}}^{\simeq}\right)=\tau$.

Example 2. Consider the language $\mathcal{L}$ of example 1. Let $\mathrm{I}^{\text {min }}$ be the minimal nteinterpretation of $\mathcal{L}$. Then $\Upsilon_{\mathbf{I} \text { min }}^{(1)}=\left\{r_{v}^{\min }, r_{\neg v}^{\min }\right\}$ is such that $\mathbf{H}\left(\mathrm{t}^{\approx}\right)=\tau_{\neg v}^{\min }$ iff $\mathrm{t}$ is not a variable, where $\mathrm{H}: \overline{\text { Terms }} \cong \Upsilon_{\text {Imin }}$.

Let $I^{\text {ex } 1}$ be the $n t e$-interpretation of example $1\left(\mathbf{I}^{\min } \preceq \mathbf{I}^{\text {ex } 1}\right.$ forms a simple ascending chain of nte-interpretations of $\mathcal{L}$ ). Then $\Upsilon_{I_{\text {ex1 }}^{(1)}}^{(1)}=\left\{\tau_{v}^{e x 1}, \tau_{\gamma}^{e x 1}, \tau_{b}^{e x 1}\right\}$. The homomorphism $H: \Phi_{I \max } \rightarrow \Phi_{I^{e x 1}}$ is such that: $H\left(\mathrm{t}^{\simeq}\right)=\tau_{\gamma}^{e x 1}$, iff the term $\mathrm{t}$ is a ground term, $\mathrm{H}(\mathrm{t} \stackrel{\simeq}{=})=\tau_{t}^{e x 1}$ iff the term $\mathrm{t}$ is a non-variable term containing a variable and $\mathrm{H}\left(\mathrm{t}^{\simeq}\right)=\tau_{t}^{e x 1}$ iff the term $\mathrm{t}$ is a variable. The $\Sigma$-algebra $\Phi_{\text {Iex } 1}$ includes identity functions with denotations $k \mapsto k, k=1,2, \ldots$. The "ground" functions (constants) with denotations $\mapsto 1$, are defined by $\varphi_{I_{\text {ex } 1}^{\mathrm{t}}=\tau_{\gamma}^{\text {ex } 1} \text {, where }}^{\mathrm{e}}$ $t$ is a ground term of $\mathcal{L}$. Functions with the denotation $1 \mapsto 1$ are defined by

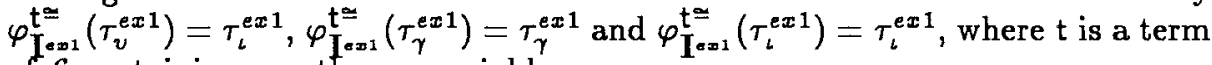
of $\mathcal{L}$ containing exactly one variable.

The homomorphism $H: \Phi_{I^{* x 1}} \rightarrow \Phi_{\text {Imin }}$ is monotonic: for instance, $H\left(\tau_{v}^{e x 1}\right)=$ 
$\tau_{v}^{\min }, \mathrm{H}\left(\tau_{\gamma}^{e x 1}\right)=\tau_{\neg v}^{\min }, \mathrm{H}\left(\tau_{\iota}^{e x 1}\right)=\tau_{\neg v}^{\min }$ and for any $\tau \in \Upsilon_{\mathbf{I}=1}^{(1)}: H\left(\varphi_{\mathbf{I} * 1}^{\mathrm{t}=}(\tau)\right)=$ $\varphi_{I \text { min }}^{\mathrm{t}=}(H(\tau))$, where $\mathrm{t}$ is a non-variable term containing one variable.

\subsection{An Abstract Domain and its Precision Theory}

Precision, or granularity, of the abstract domain $\Upsilon_{I}$ is determined by the chosen nte-interpretation $\mathbf{I}$, which determines all the properties of the corresponding abstraction function $\mathrm{H}: \overline{\text { Terms }}^{\simeq} \rightarrow \Upsilon_{\mathrm{I}}$. It can be generated as a model of the corresponding non-term equality theory by applying a model generation technique. Algorithms such as [5] are suitable. Indeed, the desired equivalence classes can be specified by means of a special first order theory $\mathcal{T}$, called a precision theory. The precision theory $\mathcal{T}$ obtained as a formalisation of a particular problem forms an input for a model generator.

In general, if there exists a finite nte-model of the precision theory $\mathcal{T}$ (i.e. a finite model of $\mathcal{T}$ with a pre-interpretation satisfying def.1), then the nte-model $\mathbf{M}_{T}^{\text {min }}$ with the minimal number of domain elements can be generated. The latter gives rise to a systematic construction of abstract interpretation algorithms, which can be tuned to fit exactly a given problem (see examples 3 and 5 ).

Example 3. The pre-interpretation of example 1 has been obtain as a pre-interpretation of the nte-model of the following precision theory $T$

for each constant $f^{(0)} \in \mathbf{F}: \operatorname{ground}(f)$

for each function $f^{(k)} \in \mathrm{F}$ :

$$
\begin{aligned}
& \forall_{X_{1}, \ldots, X_{k}} \operatorname{ground}\left(f\left(X_{1}, \ldots, X_{k}\right)\right) \leftarrow \bigwedge_{i=1}^{k} \operatorname{ground}\left(X_{i}\right) \\
& \forall_{X_{1}, \ldots, X_{k}} \operatorname{inst}\left(f\left(X_{1}, \ldots, X_{k}\right)\right) \leftarrow \bigvee_{i=1}^{k}\left(\operatorname{inst}\left(X_{i}\right) \vee \operatorname{NTE}\left(X_{i}\right)\right)
\end{aligned}
$$

$\forall X$ : $\operatorname{ground}(X) \vee$ inst $(X) \vee \operatorname{NTE}(X) \%$ Disjoint Equivalence Classes $\forall_{X}: \neg(\operatorname{ground}(X) \wedge \operatorname{inst}(X))$

where the equivalence classes $\tau_{\gamma}$ and $\tau_{\iota}$ are specified by the corresponding unary predicates ground $^{(1)}$ and inst $t^{(1)}$, respectively. The equivalence class of all variables $\tau_{v}$ is automatically specified by $\exists: \operatorname{NTE}(X)$. The elements of $\tau_{v}$ are always disjoint from the elements of any other equivalence class. Notice that $\operatorname{NTE}(X)$ is used as a "built-in" for constructing specifications of "types with variables". This precision theory, called FGI-theory (Freeness, Groundness, Instantiation), can be used for freeness, groundness and dependency analysis [1].

Our FGI-theory can be used to construct more complex types as follows. Suppose we need the type of all non-empty lists, which are constructed from variables. To do this we extend the $F G I$-theory with axioms for the new type vlist

$$
\begin{array}{ll}
\forall: v l i s t([H]) \leftarrow \mathrm{NTE}(H) & \forall: \text { inst }(X) \leftrightarrow \text { vlist }(X) \vee \text { inst_not_vl }(X) \\
\forall: v \operatorname{vist}([H \mid T] \leftarrow \mathrm{NTE}(H) \wedge \text { vlist }(T) & \forall: \neg(\text { vlist }(X) \wedge \text { inst_not_vl }(X))
\end{array}
$$


This refines the pre-interpretation $I_{0}{ }^{e x 1}$ of example 1 as follows. Firstly notice that the "ground world" represented by the first two columns of the mapping cannot be influenced by the theory above. So, a model generator is enforced to reconstruct the "non-ground world":

$$
\begin{array}{llll}
{\left[v \mid \iota_{v l}\right] \mapsto \iota_{v l}} & {[v \mid v] \mapsto \iota_{n v l}} & {\left[\gamma \mid *_{\iota}\right] \mapsto \iota_{n v l}} & s(v) \mapsto \iota_{n v l} \\
{[v \mid \gamma] \mapsto \iota_{v l}} & {\left[v \mid \iota_{n v l}\right] \mapsto \iota_{n v l}} & {\left[*_{\iota} \mid *\right] \mapsto \iota_{n v l}} & s\left(\iota_{v l}\right) \mapsto \iota_{n v l} \\
& & & s\left(\iota_{n v l}\right) \mapsto \iota_{n v l}
\end{array}
$$

where $*_{\iota}$ denotes domain elements which are $\iota_{v l}$ or $\iota_{n v l}$. The refinement is achieved by splitting out the domain element $\iota$ into $\iota_{v l}$ and $\iota_{n v}$. The domain element $\iota$ disappears. Now the non-ground primitive types have a new more refined structure: the type $\tau_{v}^{e x 3}$ of variables, the type $\tau_{\iota_{n v 1}}^{e x 3}$ of instantiated terms which are not lists of variables and the type $\tau_{\iota_{v l}}^{e x 3}$, which only includes lists of variables, i.e. it is not exactly what has been expected, e.g. the term $[X, Y, X, s([])]$ belongs to $\tau_{\iota_{v i}}^{e x 3}$. The reason is that the precision of the "ground world" is not enough to distinguish the constant [] and other ground terms (this refinement will done below, see example 5).

The nte-interpretation just above, noted $\mathrm{I}^{e x 3}$, extends the ascending chain of example 2, i.e we have $I^{\text {min }} \preceq I^{e x 1} \preceq I^{e x 3}$. The $\Sigma$-algebra $\Phi_{\text {Iex } 3}$ is a refinement of $\Phi_{I=x 1}$, i.e there exists a monotonic homomorphism $H: \Phi_{\text {Iex } 3} \rightarrow \Phi_{I=11}$ where, for instance, $\mathrm{H}\left(\tau_{v}^{e x 3}\right)=\tau_{v}^{e x 1}, \mathrm{H}\left(\tau_{\gamma}^{e x 3}\right)=\tau_{\gamma}^{e x 1}, \mathrm{H}\left(\tau_{\iota_{v l}}^{e x 3}\right)=\tau_{\iota}^{e x 1}, \mathrm{H}\left(\tau_{\iota_{n v 1}}^{e x 3}\right)=\tau_{\iota}^{e x 1}$. Now the set $\Phi_{\text {I:D3 }}$ is a bit more interesting: for instance, it includes the functions $\varphi_{[a x 3}^{[X, a]^{\cong}}$ and $\varphi_{[a x 3}^{[a, X] \cong}$ with the denotation $1 \mapsto 1$. These functions are defined by

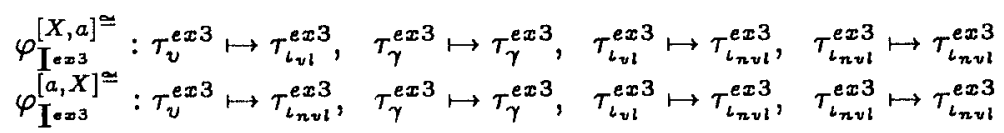

Let us summarise the most important points of the above discussion. Any nteinterpretation $I$ of the complete lattice $\mathcal{I}^{\text {nte }}(\mathcal{L})$ generates a monotonic manysorted $\Sigma$-algebra $\Phi_{\mathrm{I}}$ with domain $\Upsilon_{\mathbf{I}}$. Finite abstract domains of arbitrary precision can be constructed using a model generator. Complex domain constructions can be obtained in a iterative way as an ascending chain of nte-interpretations. This allows to fit a given problem (see example 5).

In this way the lattice $\mathcal{I}^{\text {nte }}(\mathcal{L})$ provides a complete family of abstractions of the initial monotonic algebra $\overline{\text { Terms }} \simeq$. The properties of the $\Sigma$-algebra generated by a nte-interpretation $I$ are important to describe the precision of the approximation given by the abstraction function $H: \overline{\text { Terms }} \stackrel{\simeq}{\rightarrow} \Upsilon_{\mathbf{I}}$.

\section{Abstract Interpretation for Definite Logic Programs}

\subsection{Abstracting (Syntactic) Unification}

We use a notion of constraint system which has been motivated by the generic approach suggested in $[9,12]$. Let $\mathcal{L}$ be an equality language. $\mathrm{A}$ constraint system 
based on $\mathcal{L}$, noted $\mathcal{S}$, is a pair $\langle\mathcal{C}, \mathrm{I}\rangle$, where $\mathrm{I}$ is a nte-interpretation of $\mathcal{L}$ and the set $\mathcal{C}$, called a set of constraints, is a set of well-formed formulas of $\mathcal{L}$. In the sequel we use very simple set of constraints - a constraint $\mathbf{C} \in \mathcal{C}$ (from here on constraints will be given in bold) is a formula of $\mathcal{L}$ constructed from equality atoms only using the connective $\wedge$ and the quantifier $\exists$. A constraint is said to be atomic, noted $\mathbf{A}$, iff it is a non-empty conjunction of equality atoms, i.e. it has the form $\wedge_{i=1}^{n} t_{i}=s_{i}, n>0$, where $t_{i}, s_{i}$ are terms of $\mathcal{L}$.

Consider the constraint $\left.\mathbf{A}\right|_{\bar{X}}, \bar{X} \subseteq \operatorname{var}(\mathbf{A}), \bar{X} \neq \emptyset$. Let $\mathbf{I}$ be a nte-interpretation. Suppose that there exists a tuple of terms based on $\mathcal{L}$, say $\overline{\mathrm{t}}$, such that $\mathbf{I} \models \forall:\left.\left.(\bar{X}=\overline{\mathrm{t}})\right|_{\bar{X}} \rightarrow \mathbf{A}\right|_{\bar{X}}$, where $\bar{X}$ ind $\overline{\mathrm{t}}$. Let $\tau$ be a type of $\Upsilon_{\mathbf{I}}$ such that $\overline{\mathrm{t}} \in \tau$. Then the above condition holds for any tuple of terms $\overline{\mathrm{s}}$ such that $\overline{\mathrm{s}} \in \tau$ and $\bar{s}$ ind $\bar{X}$ (cf. def. 3). The type $\tau$ satisfying these conditions is said to be a solution of the constraint $\left.\mathbf{A}\right|_{\bar{X}}$ wrt $\mathbf{I}$. The set of all solutions of $\left.\mathbf{A}\right|_{\bar{X}}$ wrt $I$ is noted $\mathbf{S}_{\mathbf{I}}\left(\left.\mathbf{A}\right|_{\bar{X}}\right) \subseteq \Upsilon_{\mathbf{I}}$.

If $\tau \in \mathrm{S}_{\mathbf{I}}\left(\left.\mathbf{A}\right|_{\bar{X}}\right)$, then any $\tau^{\prime} \in \Upsilon_{\mathbf{I}}$ such that $\tau \sqsubseteq_{\mathbf{I}} \tau^{\prime}$ is also a solution, i.e. any instance of a solution wrt $I$ is also a solution (cf. def.3). The solution $\tau \in \mathrm{S}_{\mathbf{I}}\left(\left.\mathbf{A}\right|_{\bar{X}}\right.$ ) is most general iff for any other solution $\tau^{\prime} \in \mathrm{S}_{\mathbf{I}}\left(\left.\mathbf{A}\right|_{\bar{X}}\right)$ holds: $\tau \sqsubseteq_{\mathbf{I}} \tau^{\prime}$. It is not difficult to see that there exists a most general solution $\tau \in \mathbf{S}_{\mathbf{I}}\left(\left.\mathbf{A}\right|_{\bar{X}}\right)$ iff $\mathbf{I} \models \forall:\left.\left.\quad \mathbf{A}\right|_{\bar{X}} \leftrightarrow(\bar{X}=\overline{\mathrm{t}})\right|_{\bar{X}}$ for any tuple of terms $\overline{\mathrm{t}} \in \tau$ such that $\overline{\mathrm{t}}$ ind $\bar{X}$.

A solution is said to be syntactic iff it is a solution of $\left.\mathbf{A}\right|_{\bar{X}}$ wrt the maximal nte-interpretation $\mathbf{I}^{\max }$. It is important to realize that a syntactic solution is a "projection" onto $\bar{X}$ of a unifier of $\mathbf{A}$. The most general syntactic soluticn corresponds to the most general unifier (cf. that unifiers are defined up to renaming, see $[11,17])$. The following gives a relation between syntactic solutions and solutions wrt a nte-interpretation.

\section{Definition 7. A Positive Constraint}

Consider an atomic constraint $\mathbf{A}$ based on $\mathcal{L}$ and a tuple of distinct variables $\bar{X} \neq \emptyset, \bar{X} \subseteq \operatorname{var}(\mathbf{A})$. The constraint $\left.\mathbf{A}\right|_{\bar{X}}$ is said to be positive wrt a nteinterpretation $\mathbf{I}$ of $\mathcal{L}$ iff it has a most general solution wrt $\mathbf{I}$, say $r \in \Upsilon_{\mathbf{I}}$, such that the most general syntactic solution of $\left.\mathbf{A}\right|_{\bar{X}}$, say $\overline{\mathrm{t}}^{\simeq}$, satisfies the following condition: $\overline{\mathrm{t}} \in \tau$. Otherwise $\left.\mathbf{A}\right|_{\bar{X}}$ is said to be negative.

Proposition 8. Let $\mathbf{I} \preceq \mathbf{I}^{\prime}$ be nte-interpretations of $\mathcal{L}$ such that $\mathbf{H}: \Phi_{\mathbf{I}^{\prime}} \rightarrow \Phi_{\mathbf{I}}$. Given an atomic constraint $\mathrm{A}$ and a tuple of distinct variables $\bar{X} \neq \emptyset, \bar{X} \subseteq$ $\operatorname{var}(\mathbf{A})$, holds: (1) $\mathrm{H}\left(\mathrm{S}_{\mathbf{I}^{\prime}}\left(\left.\mathbf{A}\right|_{\bar{X}}\right)\right) \subseteq \mathrm{S}_{\mathbf{I}}\left(\left.\mathbf{A}\right|_{\bar{X}}\right)^{4}$ and (2) $\mathrm{H}\left(\mathrm{S}_{\mathbf{I}^{\prime}}\left(\left.\mathbf{A}\right|_{\bar{X}}\right)\right)=\mathrm{S}_{\mathbf{I}}\left(\left.\mathbf{A}\right|_{\bar{X}}\right)$ provided that $\left.\mathrm{A}\right|_{\bar{X}}$ is positive wrt $\mathrm{I}$.

This means that the homomorphism $\mathrm{H}$ preserves solutions. In particular, any $\Sigma$-algebra provides a safe approximation of the set of syntactic solutions. In general, the most general solution is not preserved, i.e. the homomorphic image

4 The proof is obtained by applying prop. 6 to a functional equation constructed from the functions of $\Phi_{I}$ over the domain $\gamma_{I}$ which is equivalent with the constraint $A$. The idea, called "target-tupling", is sketched in [14]: e.g., $X=[a]$ is equivalent (has the same solutions wrt I) with $\varphi_{\mathbf{I}}^{\langle X, X>\cong}(\tau)=\varphi_{\mathbf{I}}^{\langle X,[a]>\stackrel{\cong}{*}}(\tau)$, where $\tau \in \Upsilon_{\mathbf{I}}^{(1)}$. 
of a most general solution is not necessarily most general. However, solutions wrt nte-interpretation of a positive constraint are exactly the homomorphic images of the syntactic solutions and the monotonicity of the homomorphism ensures that the most general syntactic solution has a most general homomorphic image.

Example 4. Let $\mathcal{L}$ be the language of example 1. The corresponding ascending chain $\mathbf{I}^{\min } \preceq \mathbf{I}^{\text {ex1 }} \preceq \mathbf{I}^{\text {ex3 }}$ of $n$ te-interpretations has been discussed in examples 2 and 3. Consider the constraints $[b, X]=[Y, a]$ and $s(X)=s(Y)$ which have the distinct syntactic most general solutions $\langle a, b\rangle \cong$ and $\langle U, U\rangle \cong$, respectively.

Let $I^{\text {min }}$ be the minimal interpretation of $\mathcal{L}$. The above constraints are equivalent wrt $I^{\mathrm{min}}$ and they have as a set of solutions the set $\Upsilon_{\text {Imin }}^{(2)}$ which has the most general element $\left.\mathrm{H}\left(<U_{1}, U_{2}\right\rangle^{\cong}\right)$ with $\mathrm{H}: \Phi_{I_{\max }} \rightarrow \Phi_{I^{e x 1}}$. However, the constraints are negative wrt $\mathrm{I}^{\mathrm{min}}$ because $\left\langle U_{1}, U_{2}{ }^{\cong}\right.$ is not a most general syntactic solution (it is too general). The above constraints are also equivalent wrt $I^{e x 1}$ having the same set of solutions, which has no most general element. The above constraints are not equivalent wrt $\mathbf{I}^{e x 3}$. However, both sets of solutions still have no most general elements.

The constraint $s(X)=s(Y) \mid X$ is positive wrt the above ascending chain of nte-interpretations because it has the most general solution $\mathrm{H}\left(\langle U\rangle^{\cong}\right)$ and $\langle U\rangle^{\cong}$ is the most general syntactic solution.

\subsection{Deriving (Computing) Properties of Definite Logic Programs}

We head now for the concepts of safe approximation and of precision of approximation for abstract interpretation of definite logic programs which are based on proposition 8 . We use ${ }^{5}$ the so-called $\mathcal{U}$-semantics which is equivalent with s-semantics (see, e.g. [2]). The $\mathcal{U}$-semantics is used in a constraint form by applying the generic approach of [12] with the syntactic notion of a unifier replaced by a solution of a constraint wrt nte-interpretation. In this way, the set $\langle\mathcal{C}, \mathbf{I}\rangle$, $\mathbf{I} \in \mathcal{I}^{\text {nte }}(\mathcal{L})$ yields a complete family of abstractions.

The syntactic $\mathcal{U}$-semantics of a constraint definite logic program $P$ (see for illustration example 5) is computed by unfolding atoms in the bodies of the clauses of $P$. This is arranged as a fixpoint operator, which produces a set of constraint facts as a fixpoint, which is $\mathcal{U}(P)$. The above discussion implies that interpreting the constraints wrt $I^{\text {max }}$ yields the syntactic $s$-success set of $P$.

Now consider $\mathbf{I} \in \mathcal{I}^{\text {nte }}$. Due to proposition $8 \mathcal{U}_{\mathbf{I}}(P)$ wrt $\mathbf{I}$ gives a safe approximation of the syntactic $\mathcal{U}$-semantics of $P$ because any constraint in the

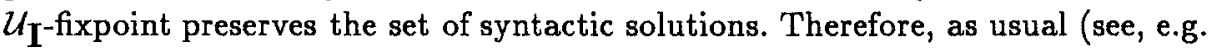
[3]), the mapping $H: \overline{\text { Terms }}^{\simeq} \rightarrow \Upsilon_{\text {I }}$ gives the concretization function as follows: if a tuple $\overline{\mathrm{t}}$ belongs to the $s$-success set of $P$ then $H\left(\overline{\mathrm{t}}^{\underline{\simeq}}\right)$ is a solution wrt $I$ of a constraint in the fixpoint. In other words, we only have negative ${ }^{6}$ information concerning properties of $P$.

\footnotetext{
The bottom-up variant of the framework is discussed in [1].

- Negative constraints cannot contain any information concerning freeness or independence of variables because these properties carry no negative information.
} 
However in our setting one can extract positive information with maximal precision provided that all constraints occurring during the fixpoint computation can be shown to be positive. The following property, called positive transitivity,

Proposition 9. Let $\mathbf{I}$ be a nte-interpretation of $\mathcal{L}$. Consider a pair of atomic constraint $\mathbf{A}^{\prime}, \mathbf{A}^{\prime \prime}$ based on $\mathcal{L}$ and a tuple of distinct variables $\bar{Y} \neq \emptyset$ such that $\bar{Y} \subseteq \operatorname{var}\left(\mathbf{A}^{\prime}\right)$ and $\bar{Y} \subseteq \operatorname{var}\left(\mathbf{A}^{\prime \prime}\right)$. Suppose that the constraints $\left.\mathbf{A}^{\prime}\right|_{\bar{Y}}$ and $\left.\mathbf{A}^{\prime \prime}\right|_{\bar{Y}}$ are positive wrt $\mathbf{I}$ and equivalent wrt $\mathbf{I}$ (i.e. $\mathbf{I} \vDash \forall:\left.\left.\mathbf{A}^{\prime}\right|_{\bar{Y}} \leftrightarrow \mathbf{A}^{\prime \prime}\right|_{\bar{Y}}$ ). Given a constraint $\mathbf{A}$ based on $\mathcal{L}$ such that $\bar{X} \cup \bar{Y} \subseteq \operatorname{var}(\mathbf{A}), \bar{X} \neq \emptyset, \bar{X}$ ind $\bar{Y}$, holds: $\left.\left(\left.\left.\mathbf{A}^{\prime}\right|_{\bar{Y}} \wedge \mathbf{A}\right|_{\bar{X} \cup \bar{Y}}\right)\right|_{\bar{X}}$ is positive wrt $\mathbf{I}$ iff $\left.\left(\left.\left.\mathbf{A}^{\prime \prime}\right|_{\bar{Y}} \wedge \mathbf{A}\right|_{\bar{X} \cup \bar{Y}}\right)\right|_{\bar{X}}$ is positive wrt $\mathbf{I}$.

ensures that if all constraints which have been involved in the fixpoint computation (i.e. the constraints of the fixpoint and all discarded redundant constraints) are positive then the most general solutions wrt $I$ of the constraints found in the fixpoint are homomorphic images of the syntactic s-success of the given program. In other words, we have a description (rather than an approximation) of the $s$-success set with the granularity of the types (equivalence classes) of $\Upsilon_{\mathbf{I}}$.

Example 5. Consider the following well-known program:

$$
\begin{aligned}
& p(X, Y) \quad \leftarrow \operatorname{rev}(X, U), \operatorname{rev}(U, Y) \diamond\{\} \\
& \operatorname{rev}(X, Y) \leftarrow \diamond\{X=[] \wedge Y=[]\} \\
& \operatorname{rev}(X, Y) \leftarrow \operatorname{rev}(T, R), \operatorname{app}(R, L, Y) \diamond\left\{\left.(X=[H \mid T] \wedge L=[H])\right|_{X, T, L}\right\} . \\
& \operatorname{app}(X, L, Y) \leftarrow \diamond\{(X=[] \wedge L=Y)\} . \\
& \operatorname{app}(X, L, Y) \leftarrow \operatorname{app}(T, L, R) \diamond\left\{\left.(X=[H \mid T] \wedge Y=[H \mid R])\right|_{X, L, Y, T, R}\right\}
\end{aligned}
$$

Suppose that we would like to prove that $p(X, Y)$ has the following property: $X$ is a list of variables iff $Y$ is a list of variables. This kind of static analysis problem is known to be difficult (see, e.g. [15]). To do this we refine the precision theory of example 3 by extending it as follows:

$$
\begin{array}{ll}
\exists: \text { elist }([]) & \forall: \operatorname{ground}(X) \leftrightarrow \text { elist }(X) \vee \text { ground_not_elist }(X) \\
& \forall: \neg(\text { elist }(X) \wedge \text { ground_not_elist }(X))
\end{array}
$$

This yields the following nte-interpretation, noted $\mathbf{I}^{e x 5}$ :

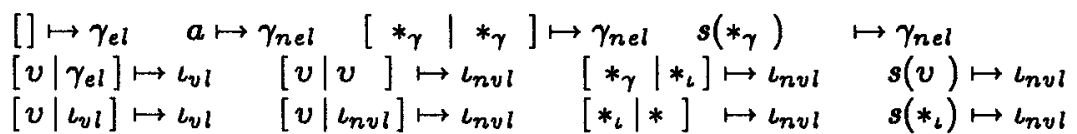

where $*_{\gamma}$ denotes $\gamma_{n e l}$ or $\gamma_{e l}\left(\gamma\right.$ of $\mathbf{I}^{e x 3}$ is split out into $\gamma_{n e l}$ and $\gamma_{e l}$ ).

The $\Sigma$-algebra $\Phi_{\text {Iexs }}$ exactly fits the problem - now the type $\tau_{l v l}^{\text {ex } 5} \in \Upsilon_{\text {Iexs }}{ }^{(1)}$ has the following property: $H\left(<t>^{\simeq}\right)=\tau_{\iota v t}^{\text {ex5 }}$ iff the term $t$ is a non-empty list constructed from variables, where $H: \Phi_{\text {Imax }} \rightarrow \Phi_{\text {Iexs }}$. Moreover, using proposition 6, one can show that: $(1)<\mathrm{t}_{x}, \mathrm{t}_{y}>\in \mathrm{H}(<[],[]>\cong)$ iff the terms $\mathrm{t}_{x}$ and $t_{y}$ are empty lists and $\left.(2)<t_{x}, t_{y}>\in H(<[U],[U]\rangle^{\cong}\right)$ iff the terms $t_{x}$ and $t_{y}$ are non-empty lists constructed from the same set of variables. 
The constraint atoms below are the $\mathcal{U}_{\text {I.as }}$ of the above program (the symbol "\%" indicates redundant (equivalent wrt $\mathbf{I}^{\text {ex } 5}$ ) elements, which are important to show that all constraints involved in the fixpoint computation are positive):

$$
\begin{aligned}
& p_{0}: p(X, Y) \diamond\{X=[] \wedge Y=[]\} \\
& p_{1}: p(X, Y) \diamond\left\{\left.\left(X=\left[H_{1}^{\prime}\right] \wedge Y=\left[H_{1}^{\prime \prime}\right] \wedge\left[H_{1}^{\prime}\right]=\left[H_{1}^{\prime \prime}\right]\right)\right|_{X, Y}\right\} \\
& \%: p(X, Y) \diamond\left\{\left(X=\left[H_{1}, H_{2}\right] \wedge Y=\left[H_{1}^{\prime \prime}, H_{2}^{\prime \prime}\right] \wedge\left[H_{2}^{\prime}, H_{1}\right]=\left[H_{1}^{\prime}, H_{1}^{\prime \prime}\right]\right.\right. \\
& \left.\left.\quad \wedge\left[H_{2}\right]=\left[H_{2}^{\prime}\right] \wedge\left[H_{1}^{\prime}\right]=\left[H_{2}^{\prime \prime}\right]\right)\left.\right|_{X, Y}\right\} \\
& r_{0}: \operatorname{rev}(X, Y) \diamond\{X=[] \wedge Y=[]\} \\
& r_{1}: \operatorname{rev}(X, Y) \diamond\left\{\left.(X=[H] \wedge Y=[H])\right|_{X, Y}\right\} \\
& \%: \operatorname{rev}(X, Y) \diamond\left\{\left.\left(X=\left[H, H^{\prime}\right] \wedge Y=\left[H^{\prime \prime}, H\right] \wedge\left[H^{\prime}\right]=\left[H^{\prime \prime}\right]\right)\right|_{X, Y}\right\} \\
& a_{0}: \operatorname{app}(X, L, Y) \diamond\{X=[] \wedge L=Y\} \\
& a_{1}: \operatorname{app}(X, L, Y) \diamond\left\{\left.(X=[H] \wedge Y=[H \mid L])\right|_{X, L, Y}\right\} \\
& \%: \operatorname{app}(X, L, Y) \diamond\left\{\left.\left(X=\left[H, H^{\prime}\right] \wedge Y=\left[H, H^{\prime} \mid L\right]\right)\right|_{X, L, Y}\right\}
\end{aligned}
$$

The above constraints were simplified only using the $E Q$-axioms and first order tautologies because this preserves their equivalence wrt any nte-interpretation.

Due to the structure $p(X, Y) \diamond \mathbf{A}^{p_{i}}, i=0,1$ of the above fixpoint, the properties of the variables $X$ and $Y$ are implied by the constraints $A^{p_{i}}, i=0,1$. The sets $\mathrm{S}_{\mathbf{I = x}}\left(\left.\mathrm{A}^{p_{0}}\right|_{X, Y}\right)$ and $\mathrm{S}_{\mathbf{I}=\mathrm{s}}\left(\left.\mathrm{A}^{p_{1}}\right|_{X, Y}\right)$ have the most general elements $\left\{H(<],\left[>^{\simeq}\right)\right\}$ and $\left\{H\left(<[U],[U]>^{\simeq}\right)\right\}$, respectively. The $s$-success set of $p^{(2)}$ (i.e. the set of tuples $\left\langle\mathrm{t}_{x}, \mathrm{t}_{y}\right\rangle$ which are computed answers for $p(X, Y)$ ) is approximated by: $\left\langle\mathrm{t}_{x}, \mathrm{t}_{y}\right\rangle \cong \in \mathrm{H}\left(\langle[],[]\rangle^{\cong}\right)$ or $\left\langle\mathrm{t}_{x}, \mathrm{t}_{y}\right\rangle^{\cong} \in \mathrm{H}\left(\langle[U],[U]\rangle^{\cong}\right)$ (i.e. $t_{x}$ and $t_{y}$ are lists constructed from the same set of variables) because all elements of the fixpoint (including redundant ones) are positive. The positive transitivity ensures that if the computation runs infinitely then all redundant constraints which will occur are positive wrt $I^{e x 5}$.

However, the precision of the abstraction function $\mathrm{H}$ is not enough to prove that $t_{x}$ and $t_{y}$ are lists of distinct variables because, for instance, $H\left([X, X]^{\simeq}\right)=$ $\mathrm{H}\left([X, Y]^{\simeq}\right)=\tau_{\iota_{v 1}}^{e x 5}$. In this particular case these lists can be made distinguishable by extending the above precision theory with the following new precision axiom $\forall_{X} \neg\left(\left.\left.\left(X=\left[Y_{1}, Y_{2}\right] \wedge \operatorname{NTE}\left(Y_{1}\right) \wedge \operatorname{NTE}\left(Y_{2}\right)\right)\right|_{X} \wedge(X=[Y, Y] \wedge \operatorname{NTE}(Y))\right|_{X}\right)$. It makes the lists $[X, X]$ and $[X, Y]$ not equivalent by introducing at least two nonterm elements and by reconstructing the non-ground world of $\mathrm{I}^{\text {ex } 5}$ (extending the above precision theory with $N T E_{2}$ does not solve the problem).

\section{Conclusion}

In our settings the abstract domain construction is a crucial step. The precision theory is supposed to be a formalisation of a hypothesis concerning expected properties of a given program. This can be considered as a generalisation of the technique of combining domains of $[7,8]$.

If the hypothesis is true, one can expect that the fixpoint only contains positive elements. In this case the analysis gives the homomorphic images of the "concrete" $s$-success set. This naturally fits the concept of an abstract data type 
of [24]. This is an important observation because it relates the algebraic specification setting and the concept of deriving properties by abstract interpretation. We expect that this relationship can be used to develop efficient compilation techniques for logic programs and that a lot of results can be adapted to develop an efficient implementation of the approach.

Existing approaches (see, e.g. $[13,3]$ ) require two concepts: an abstract domain and an abstract interpretation mechanism itself which significantly influences precision of approximation. For instance, by applying the so-called magic set transformation in the framework of [13] one can increase the precision of the bottom-up algorithm. In our setting the above top-down schema based on the $\mathcal{U}$-semantics and the bottom-up variant of [1] are equivalent and the magic set transformation can only increase efficiency, not the precision of the approximation. The reason is that the lower upper bound operation is not used in the standard way. The chosen nte-interpretation itself performs the generalisation, i.e. the lub-algorithm is "buried" in the abstract domain.

Replacing the notion of computed answer substitution by the model-theoretic setting allows a uniform treatment of a large class of static analysis problems and gives rise to a systematic abstract domain construction.

\section{Acknowledgements}

We are grateful to John Shepherdson, Marc Denecker, Bart Demoen and Gerda Janssens for extensive and important discussions on the topic of the paper.

\section{References}

1. Boulanger,D., Bruynooghe,M., Denecker,M., Abstracting s-semantics: A ModelTheoretic Approach, Proc. PLILP'94. (to appear)

2. Bossi,A., Gabrielli,M., Levi,G., Martelli,M., The s-semantics Approach: Theory and Applications, J. Logic Programming, 1994. (to appear)

3. Bruynooghe,M., Boulanger,D., Abstract Interpretation for (Constraint) Logic Programming, in: Constraint Programming, Eds. B.Mayoh, E.Tõugu, J.Penjam, NATO Advanced Sci. Series, Comput. and System Sci., Springer, 1994. (to appear)

4. Bruynooghe,M., Codish,M., Freeness, Sharing, Linearity and Correctness - All at Once, Proc. 3-rd Int. Workshop WSA'93, Springer, 1993.

5. Bry,F., Decker,H., Mathey,R., A Uniform Approach to Constraint Satisfaction and Constraint Satisfiability in Deductive Databases, Proc. Extended Database Technology 1988, Venice, LNCS, Springer, 1988.

6. Codish,M., Dams,D., File,G., Bruynooghe,M., Freeness Analysis for Logic Programs - And Correctness?, Proc. 10-th Int. Conf. Logic Prog., 1993, 116-131.

7. Codish,M., Mulkers,A., Bruynooghe,M., Garcia de la Banda,M., Hermenegildo,M., Improving Abstract interpretations by Combining Domains, Proc. ACM Symp. on Partial Evaluation and Semantics based Program Manipulation, 1993.

8. Cousot,P., Cousot,R., Systematic Design of Program Analysis Frameworks, Proc. 6-th ACM Symp. Principles Prog. Languages, San Antonio, USA, 1979, 269-282.

9. Codognet,P., File, G., Computations, Abstractions and Constraints in Logic Programs, Proc. 4-th Int. Conf. Programming Languages, Oakland, USA, 1992. 
10. Denecker,M., Knowledge Representation and Reasoning in Incomplete Logic Programming, Ph.D. Thesis, Dept. Comp. Science, K.U.Leuven, September, 1993.

11. Eder,E., Properties of Substitutions and Unifications, J. Symbolic Computation, 1985, Vol.1, No.1, 31-46.

12. Giacobazzi,R., Debray,S., Levi,G., Generalised Semantics and Abstract Interpretation for Constraint Logic Programs, J. Logic Programming, 1994. (to appear)

13. Gallagher,J., de Waal,D.A., Fast and Precise Regular Approximations of Logic Programs, Proc. 11-th Int. Conf. Logic Programming, 1994.

14. Goguen,J., Thatcher,E., Waggner,E., Wright,J., Initial Algebra Semantics and Continuous Algebras, J. ACM, 1977, Vol.24, No.1, 68-95.

15. Janssens,G., Bruynooghe,M., Deriving Descriptions of Possible Values of Program Variables by Means of Abstract Interpretation, J. Logic Programming, 1992, Vol.13, Nos.2-3, 205-258.

16. Jacobs,L., Langen,A., Static Analysis of Logic Programs for Independent AndParallelism, J. Logic Programming, 1992, Vol.13, Nos.2-3, 291-314.

17. Lassez,J.-L., Maher,M., Mariott,K., Unification Revisited, Foundations of Deductive Databases and Logic Programming, Morgan-Kaufmann, 1988, 587-625.

18. Mac Lane,S., Categories for the Working Mathematician, Springer-Verlag, 1971.

19. Lloyd,L., Foundations of Logic Programming, Springer-Verlag, Berlin, 1987.

20. Martelli,A., Montanari,U., An Efficient Unification Algorithm, Transaction on Programming Languages and Systems, 1982, Vol.2, No.4, 258-282.

21. Mariott,K. Sondergaard,H., Semantics-based Data Flow Analysis of Logic Programs, Information Processing, April 1989, 601-606.

22. Sondergaard,H., An Application of Abstract Interpretation of Logic Programs: Occur Check Reduction, LNCS, Vol.123, 1986, Springer, 327-338.

23. Shepherdson, J., Language and Equality Theory in Logic Programming, Report No.PM-88-08, School of Mathematics, Bristol, England.

24. Wirsing,W., Algebraic Specifications, in: Handbook of Theoretical Computer Science, North Holland, 1990, 678-778. 